\title{
ON SOME SUBALGEBRAS \\ OF A VON NEUMANN ALGEBRA CROSSED PRODUCT
}

BY

BARUCH SOLEL

\begin{abstract}
We study conditions for a nonselfadjoint subalgebra of a von Neumann crossed product $\mathcal{L}$ to be an algebra of analytic operators with respect to a flow on $\mathcal{L}$. We restrict ourselves to the case where $e$ is constructed from a finite von Neumann algebra $M$ with a trace preserving *-automorphism $\alpha$ that acts ergodically on the center of $M$.
\end{abstract}

1. Introduction. In [9] we studied subalgebras of a von Neumann algebra $\mathcal{L}$, constructed as a crossed product of a finite von Neumann algebra $M$ by a trace preserving *-automorphism $\alpha$, that contain the nonselfadjoint crossed product $\mathcal{L}_{+}$. With the results of $[9, \S 4]$ we can prove (see Corollary 3.5 ) that each such subalgebra is an algebra of analytic operators with respect to some flow $\beta$; i.e. it has the form $\varrho^{\beta}[0, \infty)($ see $[2])$.

In this paper we study conditions on subalgebras $\beta$ of $\mathcal{L}$ (weaker than the condition $\mathscr{B} \supseteq \mathcal{L}_{+}$) that are sufficient to ensure $\mathscr{B}$ has the form $\varrho^{\beta}[0, \infty)$ for some

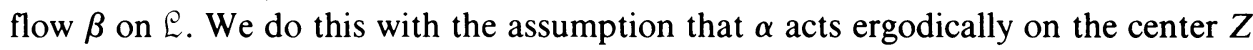
of $M$.

We deal separately with the cases $Z$ nonatomic and $Z$ atomic (in the latter case we add the assumption that $\alpha^{k}$ is outer for each $k \in \mathbf{Z}$ ). The assumptions allow us to use the results of [6].

In both cases we find (Theorem 3.4 and Corollary 3.5) sufficient conditions for the algebra to be of the form $\mathcal{L}^{\beta}[0, \infty)$. Moreover, we show how the flow $\beta$ is derived from the algebra in question.

For the case where $Z$ is atomic we also find that any subalgebra of $\mathcal{L}$ containing a subalgebra of the form $\mathcal{L}^{\beta}\left[0, \infty\right.$ ) (for a flow $\beta$ ) has the form $\mathcal{L}^{\gamma}[0, \infty$ ) (for some other flow $\gamma$ ) (Theorem 4.2).

2. Preliminaries and the definition of $\phi$. Let $M$ be a finite von Neumann algebra with a faithful and normal finite trace $\varphi$. We assume $M$ is in standard form and identify it with the von Neumann algebra of left multiplications on $L^{2}(M, \varphi)$ (see [8]). The algebra $M^{\prime}$ is its commutant on $L^{2}(M, \varphi)$. Since $M$ has a generating and

Received by the editors January 24, 1983.

1980 Mathematics Subject Classification. Primary 46L10; Secondary 46L40, 47L25.

Key words and phrases. Crossed product, nonselfadjoint subalgebra, analyticity, flow, spectral subspaces for groups of automorphisms.

'Supported by the Fund for Basic Research, administered by the Israeli Academy of Sciences and Humanities.

(C)1984 American Mathematical Society $0002-9947 / 84 \$ 1.00+\$ .25$ per page 
separating vector, $M^{\prime}$ is also finite. We write $Z$ for $M \cap M^{\prime}$ and identify it with $L^{\infty}(X, v)$ for some locally compact Hausdorff space $X$ with a probability measure $\nu$ such that

$$
\int_{X} f d \nu=\varphi(f), \quad f \in L^{\infty}(X, \nu) .
$$

We fix once and for all a normal, *-automorphism $\alpha$ of $M$ which preserves $\varphi$; i.e., $\varphi \circ \alpha=\varphi$. The following proposition appears in [4].

Proposition 2.1. Let $L_{0}^{2}=\{f: \mathbf{Z} \rightarrow M ; f(n)=0$ for all but finitely many $n\}$. Then with respect to pointwise addition, scalar multiplication and the operations defined by equations (1)-(3), $L_{0}^{2}$ is a Hilbert algebra with identity $\psi$ defined by $\psi(0)=I_{M}$ and $\psi(n)=0, n \neq 0$.

$$
\begin{aligned}
(f * g)(n) & =\sum_{k \in Z} f(k) \alpha^{k}(g(n-k)), \\
\left(f^{*}\right)(n) & =\left[\alpha^{n}(f(-n))\right]^{*}, \\
\langle f, g\rangle & =\sum_{k \in Z}(f(k), g(k))_{L^{2}(M, \varphi)} .
\end{aligned}
$$

Note that the Hilbert space completion $L^{2}$ of $L_{0}^{2}$ is

$$
\left\{f: \mathbf{Z} \rightarrow L^{2}(M, \varphi) ; \sum_{n \in \mathbf{Z}}\|f(n)\|_{L^{2}(M, \varphi)}^{2}<\infty\right\} .
$$

For $f$ in $L_{0}^{2}$ we define operators $L_{f}$ and $R_{f}$ on $L^{2}$ by $L_{f} g=f * g$ and $R_{f} g=g * f$, $g \in L^{2}$. Both $L_{f}$ and $R_{f}$ are well-defined, bounded operators, and we set $\mathcal{L}=\left\{L_{f}\right.$ : $\left.f \in L_{0}^{2}\right\}^{\prime \prime}, \Re=\left\{R_{f}: f \in L_{0}^{2}\right\}^{\prime \prime}$. Also, we define $L^{\infty}$ to be the achieved Hilbert algebra of all bounded elements in $L^{2}$. For such an $f$, we write $L_{f}$ and $R_{f}$ for the operators it determines. It is known that the map $f \rightarrow L_{f}$ [resp. $f \rightarrow R_{f}$ ] is a ${ }^{*}$-isomorphism [resp. *-anti-isomorphism] from $L^{\infty}$ onto $\mathcal{L}$ [resp. $\Re$ ]. Moreover, $\mathcal{L}$ and $\mathcal{R}$ are finite von Neumann algebras with $\Re^{\prime}=\mathfrak{L}$. We call $L^{\infty}$ the selfadjoint or von Neumann algebra

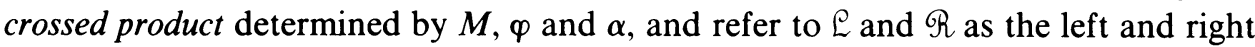
regular representations of it.

The original algebra $M$ is identified with the subalgebra $\{x \psi: x \in M\}$ of $L^{\infty}$, and we write $L_{x}$ (and $R_{x}$ ) for $L_{x \psi}$ (and $\left.R_{x \psi}\right)$. We have, for $f \in L^{2},\left(L_{x} f\right)(n)=x f(n)$ and $\left(R_{x} f\right)(n)=f(n) \alpha^{n}(x)$. We write $\mathcal{L}(M)=\left\{L_{x}: x \in M\right\}$ and $\mathcal{R}(M)=\left\{R_{x}\right.$ : $x \in M\}$.

If we let $\delta$ be defined by $\delta(n)=0$ if $n \neq 1, \delta(1)=I_{M}$, then it is easy to check that $\mathcal{L}$ is the von Neumann algebra generated by $\mathcal{L}(M)$ and $L_{\delta}$ and, similarly, $R$ is generated by $R(M)$ and $R_{\delta}$.

The automorphism group $\left\{\hat{\alpha}_{t}\right\}_{t \in \mathbf{R}}$ of $\mathcal{E}$ dual to $\alpha$ in the sense of Takesaki [10] is implemented by the unitary representation of $\mathbf{R},\left\{W_{t}\right\}_{t \in \mathbf{R}}$, defined by

$$
\left(W_{t} f\right)(n)=e^{2 \pi i n t} f(n), \quad f \in L^{2} ;
$$

that is, $\hat{\alpha}_{t}\left(L_{f}\right)=W_{t} L_{f} W_{t}^{*}$. Similarly, $\hat{\alpha}_{t}\left(R_{f}\right)=W_{t} R_{f} W_{t}^{*}$. It is easy to see that $\hat{\alpha}_{t}\left(L_{f}\right)=L_{W_{t} f}$ for $f$ in $L^{\infty}$, and similarly for $R_{f}$. One can check that the spectral 
resolution of $\left\{W_{t}\right\}_{t \in \mathbf{R}}$ is given by

$$
W_{t}=\sum_{n=-\infty}^{\infty} e^{2 \pi i n t} E_{n},
$$

where $E_{n}$ is the projection on $L^{2}$ defined by

$$
\left(E_{n} f\right)(k)= \begin{cases}f(n), & k=n, \\ 0, & k \neq n .\end{cases}
$$

We denote the restriction of $E_{n}$ to $L^{\infty}$ by $\varepsilon_{n}$ and write $\varepsilon_{n}\left(L_{f}\right)=L_{\varepsilon_{n}(f)}$. We have

$$
\varepsilon_{n}=\int_{0}^{1} e^{-2 \pi i n t} \hat{\alpha}_{t} d t
$$

where the integral converges in the $\sigma$-weak operator topology.

REMARK 2.1. If $f$ lies in $L^{\infty}$, then $\varepsilon_{k}\left(L_{f}\right)=L_{f(k)} L_{\delta}^{k}$ (see [9, Remark preceding Theorem 4.5]) for each $k \in \mathbf{Z}$. Hence,

$$
\begin{aligned}
\varepsilon_{k}\left(L_{f}^{*}\right)^{*} & =\varepsilon_{k}\left(L_{f^{*}}\right)^{*}=\left(L_{f^{*}(k)} L_{\delta}^{k}\right)^{*}=\left(L_{\alpha^{k}(f(-k))^{*}} L_{\delta}^{k}\right) * \\
& =\left(L_{\delta}^{k} L_{f(-k)^{*}}\right)^{*}=L_{f(-k)} L_{\delta}^{-k}=\varepsilon_{-k}\left(L_{f}\right) .
\end{aligned}
$$

Thus $\varepsilon_{k}\left(L_{f}^{*}\right)=\left(\varepsilon_{-k}\left(L_{f}\right)\right)^{*}$.

We let $H^{2}$ be $\left\{f \in L^{2}: f(n)=0, n<0\right\}$, and $H^{\infty}$ be $\left\{f \in L^{\infty}: f(n)=0, n<0\right\}$.

More details concerning these algebras can be found in [4 and 5].

We will define an $\mathcal{L}(Z)$-trace following [1, Chapter III, §4]. First we let $\mathscr{Z}$ be the set of all nonnegative measurable functions, finite or not, on $X$, and $\mathscr{Z}^{\prime}$ the set of all real valued (finite) measurable functions on $X$ (we identify two functions in $\mathscr{Z}$, or $\mathscr{Z}^{\prime}$, if they are different only on a set of measure zero). We identify the bounded functions in $\mathscr{Z}^{\prime}$ with $\mathscr{L}(Z)$. Hence $\mathscr{L}(Z) \subseteq \mathscr{Z}^{\prime}$ and $\mathscr{L}(Z)_{+} \subseteq \mathscr{Z}$.

Definition. An $\mathcal{L}(Z)$-trace on $\mathcal{L}(M)_{+}^{\prime}$ (the positive cone of the commutant of $\mathcal{L}(M)$ ) is a map $\phi$ defined on $\mathscr{L}(M)_{+}^{\prime}$, with values in $\mathscr{Z}$ and satisfying:

(1) If $T, S \in \mathcal{L}(M)_{+}^{\prime}$, then $\phi(S+T)=\phi(S)+\phi(T)$;

(2) If $S \in \mathcal{L}(M)_{+}^{\prime}, T \in \mathcal{L}(Z)_{+}$, then $\phi(T S)=T \phi(S)$; and

(3) If $S \in \mathcal{L}(M)_{+}^{\prime}$, and $U \in \mathcal{L}(M)^{\prime}$ is a unitary operator, then $\phi\left(U S U^{*}\right)=\phi(S)$.

$\phi$ is semifinite if for every $S \neq 0$ in $\mathcal{L}(M)_{+}^{\prime}$, there is an operator $T \in \mathcal{L}(M)_{+}^{\prime}$, $T \neq 0$, such that $T \leqslant S$ and $\phi(T) \in \mathcal{L}(Z)_{+} \cdot \phi$ is faithful if $\phi(T)=0$ only when $T=0$, and $\phi$ is normal if, for each increasing net $\left\{S_{\alpha}\right\} \subseteq \mathcal{L}(M)_{+}^{\prime}, \operatorname{Sup}_{\alpha} \phi\left(S_{\alpha}\right)=$ $\phi\left(\operatorname{Sup}_{\alpha} S_{\alpha}\right)$.

It is shown in [9] that there is a unique, faithful, normal semifinite $\mathcal{L}(Z)$-trace that maps $E_{0}$ into $I$. Henceforth, we let $\phi$ be this $\mathcal{L}(Z)$-trace.

REMARK 2.2. Consider $\alpha$ as acting on Z. By a theorem of Mackey [3] there is a measurable transformation $\tau$ on $X$ implementing $\alpha$; i.e. $\alpha(f)(x)=f(\tau(x))$. Using this we can extend the action of $\alpha$ to both $\mathscr{Z}$ and $\mathscr{Z}^{\prime}$. We claim that $\phi\left(L_{\delta} T L_{\delta}^{*}\right)=$ $\alpha(\phi(T))$ for $T \in \mathcal{L}(M)^{\prime}$ with $\phi(T)$ in $\mathcal{Z}$. Indeed, let $\phi_{1}$ be defined on $\mathcal{L}(M)_{+}^{\prime}$ by $\phi_{1}(T)=\alpha^{-1}\left(\phi\left(R_{\delta}^{*} L_{\delta} T L^{*} R_{\delta}\right)\right)$; then $\phi_{1}$ is a faithful, normal, semifinite $\mathcal{L}(Z)$-trace that maps $E_{0}$ into $I$ (because $\phi$ has these properties and $R_{\delta}^{*} L_{\delta} E_{0} L_{\delta}^{*} R_{\delta}=E_{0}$ ). Since $\phi$ is unique with these properties, the claim follows. 
Finally, we recall that if $\left\{\beta_{t}\right\}_{t \in \mathbf{R}}$ is a group of ${ }^{*}$-automorphisms on $\mathcal{L}$ such that $t \rightarrow \beta_{t}(T)$ is $\sigma$-weakly continuous for each $T \in \mathcal{L}$, then $\mathcal{L}^{\beta}[0, \infty)$ is the spectral subspace associated with $[0, \infty) \subseteq \mathbf{R}$. Such a group $\beta$ will be called a flow and $\mathcal{L}^{\beta}[0, \infty)$ is a $\sigma$-weakly closed subalgebra of $\mathcal{L}$ (see [2] for more details). We also refer to $\mathcal{L}^{\beta}[0, \infty)$ as the algebra of analytic operators with respect to the flow $\beta$.

3. Subalgebras of $\mathcal{L}$ when $\mathcal{L}(Z)$ is nonatomic. In the section and the next we frequently refer to the following four conditions, where $\mathscr{B}$ is a $\sigma$-weakly closed subalgebra of $\varrho$.

(i) $\mathscr{B}+\mathfrak{B}^{*}$ is $\sigma$-weakly dense in $\mathcal{E}$.

(ii) $\mathcal{L}(M) \subseteq \mathscr{\Re}$.

(iii) For $f \in L^{\infty}, f$ lies in $[\Re]_{2}$ ( $=$ the closure of the subspace $\{T \psi: T \in \mathscr{G}\}$ ) if and only if $L_{f} \in \mathscr{B}$.

(iv) $\phi\left(P-P L_{\delta} P L_{\delta}^{*}\right)$ and $\phi\left(L_{\delta} P L_{\delta}^{*}-P L_{\delta} P L_{\delta}^{*}\right)$ are finite a.e., where $P$ is the projection on $[\Re]_{2}$. (We shall refer to this condition only when it is known that $P$ and $L_{\delta} P L_{\delta}^{*}$ commute.)

We write $P(\mathscr{B})$ for the projection onto $[\Re]_{2}$.

Throughout this section we assume $\mathcal{L}(Z)$ is nonatomic and $\alpha$ acts ergodically on $\mathcal{L}(Z)$

LEMMA 3.1. If $\mathscr{B}$ satisfies conditions (i)-(iii) there is a sequence $\left\{e_{k}\right\}_{k=-\infty}^{\infty}$ of projections in $\mathcal{L}(Z)$ such that $P(\mathscr{B})=\sum_{k=-\infty}^{\infty} e_{k} E_{k}$ and

$$
\mathscr{B}=\left\{T \in \mathcal{L}: \varepsilon_{k}(T) \in e_{k} \mathfrak{L}(M) L_{\delta}^{k} \text { for each } k \in \mathbf{Z}\right\} .
$$

Moreover, for each $k, n \in \mathbf{Z}$, we have

$$
\begin{gathered}
e_{n} \alpha^{n}\left(e_{k}\right) \leqslant e_{n+k}, \\
1-\alpha^{-n}\left(e_{n}\right) \leqslant e_{-n}, \\
e_{n}\left(1-\alpha^{k}\left(e_{n-k}\right)\right) \leqslant e_{k} .
\end{gathered}
$$

Proof. Let $P$ be $P(\mathscr{B})$, then since $\mathcal{L}(M) \subseteq \mathscr{T}, P \in\{\mathscr{L}(M), \mathscr{R}(M)\}^{\prime}$. Using [6, Proposition 3.1] there is a sequence $\left\{e_{k}\right\}_{k=-\infty}^{\infty}$ of projections in $\mathscr{L}(Z)$ such that $P=\sum_{k=-\infty}^{\infty} e_{k} E_{k}$. (iii) now implies $\mathscr{B}=\left\{T \in \mathcal{L}: \varepsilon_{k}(T) \in e_{k} \mathcal{L}(M) L_{\delta}^{k}\right.$ for each $\left.k \in \mathbf{Z}\right\}$ since $\varepsilon_{k}(T)=L_{E_{k}(f)}$ where $T=L_{f}, f \in L^{\infty}$.

For each $k, n \in \mathbf{Z}, e_{n} L_{\delta}^{n}$ and $e_{k} L_{\delta}^{k}$ lie in $\mathscr{B}$; hence, $e_{n} \alpha^{n}\left(e_{k}\right) L_{\delta}^{n+k}=e_{n} L_{\delta}^{n} e_{k} L_{\delta}^{k} \in \mathscr{G}$ and (1) follows. For (2) note that, since $\varepsilon_{k}\left(L^{*}\right)=\left(\varepsilon_{-k}\left(L_{f}\right)\right)^{*}$ (see Remark 2.1), $\varepsilon_{k}\left(T^{*}\right) \in e_{k} \mathcal{L}(M) L_{\delta}^{k}$ for $T \in \mathcal{L}$ if and only if

$\varepsilon_{-k}(T) \in\left(e_{k} \mathcal{L}(M) L_{\delta}^{k}\right)^{*}=L_{\delta}^{-k} e_{k} \mathcal{L}(M)=L_{\delta}^{-k} e_{k} L_{\delta}^{-k} \mathcal{L}(M) L_{\delta}^{k}=\alpha^{-k}\left(e_{k}\right) \mathcal{L}(M) L_{\delta}^{k}$.

Hence,

$$
\begin{aligned}
\mathscr{B}^{*} & =\left\{T: T^{*} \in \mathscr{B}\right\}=\left\{T \in \mathcal{L}: \varepsilon_{k}\left(T^{*}\right) \in e_{k} \mathcal{L}(M) L_{\delta}^{k} \text { for each } k \in \mathbf{Z}\right\} . \\
& =\left\{T \in \mathcal{L}: \varepsilon_{k}(T) \in \alpha^{k}\left(e_{-k}\right) \mathcal{L}(M) L_{\delta}^{-k} \text { for each } k \in \mathbf{Z}\right\},
\end{aligned}
$$

and

$$
\mathscr{B}+\mathscr{B}^{*} \subseteq\left\{T \in \mathcal{L}: \varepsilon_{k}(T) \in\left(e_{k} \vee \alpha^{k}\left(e_{-k}\right)\right) \mathcal{L}(M) L_{\delta}^{k} \text { for each } k \in \mathbf{Z}\right\} .
$$


Since $\mathscr{B}+\mathscr{B}^{*}$ is $\sigma$-weakly dense in $\varrho$ and the set on the right-hand side is $\sigma$-weakly closed (as $\varepsilon_{k}$ are $\sigma$-weakly continuous), $e_{k} \vee \alpha^{k}\left(e_{-k}\right)=1$ and (2) follows.

As a consequence we have, for $n, k \in \mathbf{Z}$,

$$
e_{n}\left(1-\alpha^{k}\left(e_{n-k}\right)\right)=e_{n} \alpha^{n}\left(1-\alpha^{k-n}\left(e_{n-k}\right)\right) \leqslant e_{n} \alpha^{n}\left(e_{n-k}\right) \leqslant e_{k} ;
$$

hence, (3) holds.

Let $f$ lie in $Z^{\prime}$ and let the sequence $\left\{f_{n}\right\}_{n=-\infty}^{\infty}$ of elements of $\mathscr{Z}^{\prime}$ be defined by

$$
f_{n}= \begin{cases}\sum_{k=0}^{n-1} \alpha^{k}(f), & n>0, \\ 0, & n=0, \\ -\alpha^{n}\left(f_{-n}\right), & n<0 .\end{cases}
$$

Let $U_{t}^{(n)}$ be the unitary operator in $\mathcal{L}(Z)$ defined by

$$
U_{t}^{(n)}=\exp \left(i t f_{n}\right), \quad n \in \mathbf{Z}, t \in \mathbf{R} .
$$

Since $E_{n} E_{m}=0$ for $n \neq m$ and $U_{t}^{(n)} E_{n}\left(L^{2}\right) \subseteq E_{n}\left(L^{2}\right), U_{t}=\sum_{n=-\infty}^{\infty} U_{t}^{(n)} E_{n}$ is a unitary operator on $L^{2}$. Moreover, $t \rightarrow U_{t}$ is a strongly continuous representation of $\mathbf{R}$ (since $t \rightarrow U_{t}^{(n)}$ is strongly continuous for each $n \in \mathbf{Z}$ ). We let $\left\{\boldsymbol{\beta}_{t}\right\}_{t \in \mathbf{R}}$ be the group of *-automorphisms on $\mathcal{L}$ defined by

$$
\beta_{t}(T)=U_{t} T U_{t}^{*}, \quad T \in \mathcal{L} .
$$

We call $\left\{U_{t}\right\}_{t \in \mathbf{R}}$ the group of unitary operators and $\left\{\boldsymbol{\beta}_{t}\right\}_{t \in \mathbf{R}}$ the group of *-automorphisms given rise to by $f$ (in $\mathscr{Z}^{\prime}$ ).

A calculation similar to $[4$, p. 390$]$ reveals

$$
\beta_{t}\left(L_{g}\right)=L_{U_{t} g}, \quad t \in \mathbf{R}, L_{g} \in \mathcal{L} .
$$

There is an obvious correspondence between projections in $\mathcal{L}(Z)$ and measurable subsets of $X$ (where the projection $e$ that corresponds to $\hat{e} \subseteq X$ is of the form $L_{h}$, and $h$ is the characteristic function of $\hat{e}$ viewed as an element of $Z \simeq L^{\infty}(X, \nu)$ ).

LEMMA 3.2. Let $f$ (in $\mathscr{Z}^{\prime}$ ) give rise to a group of ${ }^{*}$-automorphisms $\left\{\beta_{t}\right\}_{t \in \mathbf{R}}$. Then $t \rightarrow \beta_{t}(T)$ is $\sigma$-weakly continuous for each $T \in \mathcal{L}$ and the algebra $\mathcal{L}^{\beta}[0, \infty)$ of analytic operators is the set $\left\{T \in \mathcal{L}: \varepsilon_{k}(T) \in c_{k} \mathscr{L}(M) L_{\delta}^{k}\right.$ for each $\left.k \in \mathbf{Z}\right\}$, where $c_{k}$ is the projection in $\mathcal{L}(Z)$ corresponding to the set $\hat{c}_{k}=\left\{x \in X: f_{k}(x) \geqslant 0\right\}$. Therefore $\complement^{\beta}[0, \infty)$ satisfies conditions (i)-(iii).

Proof. For $T \in \mathcal{L}, t \rightarrow \beta_{t}(T)=U_{t} T U_{t}^{*}$ is $\sigma$-weakly continuous since $t \rightarrow U_{t}$ is strongly continuous.

Let $P\left(P_{n}\right)$ be the spectral measure associated with the group $\left\{U_{t}\right\}_{t \in \mathbf{R}}\left(\left\{U_{t}^{(n)}\right\}_{t \in \mathbf{R}}\right.$, $n \in \mathbf{Z})$ by Stone's Theorem.

Since $U_{t}=\sum_{n=-\infty}^{\infty} U_{t}^{(n)} E_{n}$ and $U_{t}^{(n)} E_{n}\left(L^{2}\right) \subseteq E_{n}\left(L^{2}\right)$ for all $n \in \mathbf{Z}, t \in \mathbf{R}$, we have

$$
P[s, \infty)=\sum_{n=-\infty}^{\infty} P_{n}[s, \infty) E_{n} \quad \text { for each } s \in \mathbf{R} \text {. }
$$


Since $U_{t}^{(n)}$ lies in $\mathcal{L}(Z), P_{n}[s, \infty) \in \mathcal{L}(Z)$ and, in fact, $P_{n}[s, \infty)$ is the projection, in $\mathcal{L}(Z)$, corresponding to the subset $\hat{c}_{n}^{(s)}=\left\{x \in X: f_{n}(x) \geqslant s\right\}$ of $X$ (since $U_{t}^{(n)}=$ $\left.\exp \left(i t f_{n}\right)\right)$. Hence, $P[0, \infty)=\sum c_{n} E_{n}$, where $c_{n}$ corresponds to $\hat{c}_{n}=\left\{x \in X: f_{n}(x) \geqslant\right.$ $0\}$.

We now wish to show that $g$, in $L^{\infty}$, lies in $P[0, \infty)\left(L^{2}\right)$ if and only if $L_{g}$ lies in $\mathfrak{L}^{\beta}[0, \infty)$.

Let $L_{g}$ be in $\mathcal{L}^{\beta}[0, \infty)$. Then by [2, Theorem 2.9], $L_{g} P[0, \infty)\left(L^{2}\right) \subseteq P[0, \infty)\left(L^{2}\right)$. But $\psi \in P[0, \infty)\left(L^{2}\right)$ (since $P_{0}[0, \infty)=I$ and $\psi(0)=I, \psi(n)=0, n \neq 0$ ) and $L_{g} \psi=g$; hence, $g \in P[0, \infty)\left(L^{2}\right)$.

For the converse note that, since $L^{1}(T)$ has an approximate identity consisting of trigonometric polynomials, say $\left\{k_{n}\right\}_{n=1}^{\infty}$, each $T \in \mathcal{L}$ is the $\sigma$-weak limit of (finite) linear combinations of $\left\{\varepsilon_{k}(T)\right\}_{k=-\infty}^{\infty}$ (namely $\left.\int_{\pi} \hat{\alpha}_{t}(T) k_{n}(t) d t\right)$. Hence, it suffices to prove, for $g$ in $L^{\infty} \cap P[0, \infty)\left(L^{2}\right)$, that $\varepsilon_{k}\left(L_{g}\right)$ lies in $\mathcal{L}^{\beta}[0, \infty)$ for each $k \in \mathbf{Z}$.

As previously noted (Remark 2.1), $\varepsilon_{k}\left(L_{g}\right)=L_{g(k)} L_{\delta}^{k}$, hence, we now fix $k$ and prove that

$$
L_{g(k)} L_{\delta}^{k} P[s, \infty)\left(L^{2}\right) \subseteq P[s, \infty)\left(L^{2}\right) \text { for all } s \in \mathbf{R} .
$$

This will imply that $\varepsilon_{k}\left(L_{g}\right) \in \mathfrak{L}^{\beta}[0, \infty)$ (by Theorem 2.9 of [2]). Since $g \in$ $P[0, \infty)\left(L^{2}\right), E_{k}(g) \in P_{k}[0, \infty)\left(L^{2}\right)$, and if we let $p_{k}$ be the projection in $Z$ with $L_{p_{k}}=P_{k}[0, \infty)$, then $g(k)=p_{k} g(k)$ and $L_{g(k)}=P_{k}[0, \infty) L_{g(k)}$. Fix $s \in \mathbf{R}$ and $h$ in $P[s, \infty)\left(L^{2}\right)$. Then for $n \in \mathbf{Z}$,

$$
\begin{aligned}
E_{n}\left(\varepsilon_{k}\left(L_{g}\right) h\right) & =E_{n} L_{g(k)} L_{\delta}^{k} h=L_{g(k)} E_{n} L_{\delta}^{k} h=L_{g(k)} L^{k} E_{n-k} h \\
& \in L_{g(k)} L_{\delta}^{k} P_{n-k}[s, \infty) E_{n-k}\left(L^{2}\right)=L_{g(k)} \alpha^{k}\left(P_{n-k}[s, \infty)\right) E_{n}\left(L^{2}\right) \\
& \subseteq P_{k}[0, \infty) \alpha^{k}\left(P_{n-k}[s, \infty)\right) E_{n}\left(L^{2}\right) .
\end{aligned}
$$

But, from the definition of the functions $\left\{f_{k}\right\}$ in $\mathscr{Z}^{\prime}$, if $f_{k}(x) \geqslant 0$ and $\alpha^{n}\left(f_{n-k}\right)(x) \geqslant s$, then $f_{n}(x) \geqslant s$. Hence, $\hat{c}_{k} \cap \alpha^{n}\left(\hat{c}_{n-k}^{(s)}\right) \subseteq \hat{c}_{n}^{(s)}$ and $P_{k}[0, \infty) \alpha^{k}\left(P_{n-k}[s, \infty)\right) \subseteq$ $P_{n}[s, \infty)$. It follows that, for each $n \in \mathbf{Z}$,

$$
E_{n}\left(\varepsilon_{k}\left(L_{g}\right) h\right) \in P_{n}[0, \infty) E_{n}\left(L^{2}\right) \subseteq P[s, \infty)\left(L^{2}\right) .
$$

Therefore, $\varepsilon_{k}\left(L_{g}\right) h \in P[s, \infty)\left(L^{2}\right)$ and this completes the proof that $L_{g} \in$ $e^{\beta}[0, \infty)$. We conclude that

$$
\begin{aligned}
\mathcal{L}^{\beta}[0, \infty) & =\left\{L_{g} \in \mathcal{L}: g \in P[0, \infty)\left(L^{2}\right)\right\} \\
& =\left\{L_{g} \in \mathcal{L}: E_{n}(g) \in c_{n} E_{n}\left(L^{2}\right) \text { for each } n \in \mathbf{Z}\right\} \\
& =\left\{L_{g} \in \mathcal{L}: \varepsilon_{n}\left(L_{g}\right) \in c_{n} \mathcal{L}(M) L_{\delta}^{n} \text { for each } n \in \mathbf{Z}\right\} .
\end{aligned}
$$

Now $\mathscr{L}(M) \subseteq \mathfrak{L}^{\beta}[0, \infty)$ since $c_{0}=I$. Condition (i) is satisfied because of Theorem 3.15 in [2]. Condition (iii) follows from the fact that $\mathcal{L}^{\beta}[0, \infty)$ is $\left\{L_{g} \in \mathcal{L}\right.$ : $\left.g \in P[0, \infty)\left(L^{2}\right)\right\}$, because $\left[\complement^{\beta}[0, \infty)\right]_{2} \subseteq P[0, \infty)\left(L^{2}\right)$.

LemMa 3.3. Let $\mathscr{B}$ be a subalgebra of 2 satisfying (i)-(iv) and let $P$ be $P(\mathscr{B})$. For $k \in \mathbf{Z}$ let $g_{1}$ be $\phi\left(P-P L_{\delta}^{k} P L_{\delta}^{-k}\right)$ and $g_{2}$ be $\phi\left(L_{\delta}^{k} P L_{\delta}^{-k}-P L_{\delta}^{k} P L_{\delta}^{-k}\right)$. Then:

(1) $g_{1}-g_{2}=f_{k}$, where $f=\phi\left(P-P L_{\delta} P L_{\delta}^{*}\right)-\phi\left(L_{\delta} P L_{\delta}^{*}-P L_{\delta}^{*}\right) \in \mathscr{Z}^{\prime}$;

(2) $g_{1} g_{2}=0$. 
Proof. First note that $P$ commutes with $L_{\delta}^{k} P L_{\delta}^{-k}(k \in \mathbf{Z})$ because $P=$ $\sum_{n=-\infty}^{\infty} e_{n} E_{n}, L_{\delta}^{k} P L_{\delta}^{-k}=\sum_{n=-\infty}^{\infty} \alpha^{k}\left(e_{n}\right) E_{n+k}$ and $e_{n} \in \mathcal{L}(Z)$ (Lemma 3.1).

We can extend the definition of $\phi$ to all operators $T \in \mathcal{L}(M)^{\prime}$ that can be written as $T=T_{1}-T_{2}$, where $T_{1}, T_{2} \in \mathcal{L}(M)_{+}^{\prime}$ and $\phi\left(T_{i}\right), \phi\left(T_{2}\right) \in \mathscr{Z}^{\prime}$, simply by $\bar{\phi}(T)=$ $\phi\left(T_{1}\right)-\phi\left(T_{2}\right)$. Since (iv) is satisfied,

$$
f=\phi\left(P-P L_{\delta} P L_{\delta}^{*}\right)-\phi\left(L_{\delta} P L_{\delta}^{*}-P L_{\delta} P L_{\delta}^{*}\right)=\bar{\phi}\left(P-L_{\delta} P L_{\delta}^{*}\right) .
$$

Remark 2.2 can be seen to hold for $\bar{\phi}$ in place of $\phi$, thus

$$
\alpha^{n}(f)=\bar{\phi}\left(L_{\delta}^{n} P L_{\delta}^{-n}-L_{\delta}^{n+1} P L_{\delta}^{-n-1}\right),
$$

and, for $k>0$,

$$
f_{k}=\sum_{k=0}^{n-1} \alpha^{n}(f)=\bar{\phi}\left(P-L_{\delta}^{k} P L_{\delta}^{-k}\right)
$$

while for $k<0$,

$$
f_{k}=-\alpha^{k}\left(f_{-k}\right)=-\alpha^{k}\left(\phi\left(P-L_{\delta}^{-k} P L_{\delta}^{k}\right)\right)=\bar{\phi}\left(P-L_{\delta}^{k} P L_{\delta}^{-k}\right) .
$$

Thus

$f_{k}=\bar{\phi}\left(P-L_{\delta}^{k} P L_{\delta}^{-k}\right)=\phi\left(P-P L_{\delta}^{k} P L_{\delta}^{-k}\right)-\phi\left(L_{\delta}^{k} P L_{\delta}^{-k}-P L_{\delta}^{k} P L_{\delta}^{-k}\right)=g_{1}-g_{2}$.

For (2), note that $P-L_{\delta}^{k} P L_{\delta}^{-k}=\sum d_{n} E_{n}$, where $d_{n}=e_{n}\left(1-\alpha^{k}\left(e_{n-k}\right)\right) \leqslant e_{k}$ (see Lemma 3.1). Hence,

$$
g_{1}=\phi\left(\sum d_{n} E_{n}\right)=\Sigma \phi\left(d_{n} E_{n}\right)=\Sigma d_{n} \text { and } g_{1} e_{k}=g_{1} \text {. }
$$

On the other hand, $L_{\delta}^{k} P L_{\delta}^{-k}-P L_{\delta}^{k} P L_{\delta}^{-k}=\Sigma c_{n} E_{n}$, where

$$
c_{n}=\alpha^{k}\left(e_{n-k}\right)\left(1-e_{n}\right)=\alpha^{k}\left(e_{n-k}\left(1-\alpha^{-k}\left(e_{n}\right)\right)\right) \leqslant \alpha^{k}\left(e_{-k}\right) \leqslant 1-e_{k} ;
$$

thus $g_{2}=g_{2}\left(1-e_{k}\right)$ and $g_{1} g_{2}=0$.

THEOREM 3.4. Let $\mathfrak{B}$ be a $\sigma$-weakly closed subalgebra of $\mathcal{L}$ satisfying (i)-(iv). Then $\Re=\mathfrak{L}^{\beta}[0, \infty)$ for some flow $\beta$ on $\mathcal{L}$. In fact $\beta$ is the group introduced in the discussion preceding Lemma 3.2 for

$$
f=\phi\left(P-P L_{\delta} P L_{\delta}^{*}\right)-\phi\left(L_{\delta} P L_{\delta}^{*}-P L_{\delta} P L_{\delta}^{*}\right) \in \mathscr{Z}^{\prime}
$$

(where $P=P(\Re)$ ).

Proof. Keeping the notation of Lemmas 3.I and 3.2, it suffices to show that $c_{k}=e_{k}$ for each $k \in \mathbf{Z}$.

$e_{k} \leqslant c_{k}$. Let $e$ be the projection $e_{k}-e_{k} c_{k} \in \mathcal{L}(Z)$. Since $e \leqslant 1-c_{k}, f_{k}<0$ a.e. on $\hat{e}$ (the subset of $X$ that corresponds to $e$ ). Using the notation of Lemma 3.3, $f_{k}=-g_{2}$ on $\hat{e}$ (since $g_{1} g_{2}=0, f_{k}=g_{1}-g_{2}$ ). If $e \neq 0$, we have $g_{2} e \neq 0$, but we saw, in the proof of Lemma 3.3, that $g_{2}=g_{2}\left(1-e_{k}\right)$, and from the definition of $e$, $e \leqslant e_{k}$. This is a contradiction and it proves that $e_{k} \leqslant c_{k}$ for all $k \in \mathbf{Z}$.

$c_{k} \leqslant e_{k}$. Let $c$ be the projection $c_{k}\left(1-e_{k}\right)$. Since $c_{k} \leqslant c_{k}, f_{k} \geqslant 0$ a.e. on $\hat{c}$ and $g_{2} c=0$ (in the notations of Lemma 3.3). But $g_{2}=g_{2}\left(1-e_{k}\right)$ and $e \leqslant 1-e_{k}$; hence $c=0$ and $c_{k} \leqslant e_{k}$. 
COROLlaRY 3.5. Let 9 be a $\sigma$-weakly closed proper subalgebra of $\mathcal{L}$ satisfying (i)-(iii). If in addition, $\mathscr{G}$ contains $L_{\delta}^{n} \mathcal{L}_{+}$for some $n \in \mathrm{Z}$, it satisfies (iv) and, consequently, has the form $\mathcal{L}^{\beta}[0, \infty)$ for some flow $\beta$ on $\mathcal{L}$.

Proof. Let $P$ be $P(\mathscr{B})$.

To prove (iv), let $\left\{e_{k}\right\}_{k=-\infty}^{\infty}$ be the sequence of projections introduced in Lemma 3.1. If $\phi\left(L_{\delta} P L_{\delta}^{*}-L_{\delta} P L_{\delta}^{*} P\right)$ is not finite, then it is infinite everywhere on a subset $\hat{e}$ of $X$ with a corresponding projection $e \in \mathcal{L}(Z)$. Since $\phi\left(L_{\delta} P L_{\delta}^{*}-L_{\delta} P L_{\delta}^{*} P\right)=$ $\sum \alpha\left(e_{k-1}\right)\left(1-e_{k}\right)$, we have

$$
\sum \alpha\left(e_{k-1}\right)\left(1-e_{k}\right) e=\infty \cdot e
$$

and

$$
\sum e_{k-1}\left(1-\alpha^{-1}\left(e_{k}\right)\right) \alpha^{-1}(e)=\infty \cdot \alpha^{-1}(e) .
$$

We first show that, for each $k \in \mathbf{Z}, \alpha^{-1}(e) \leqslant e_{k}$. Suppose $\alpha^{-1}(e) \leqslant e_{k_{0}}$ for some $k_{0} \in \mathbf{Z}$. Then $c=\alpha^{-1}(e)\left(1-e_{k_{0}}\right) \neq 0$. Since $L_{\delta}^{n} L_{+} \subseteq \mathscr{R}, e_{m}=1$ for each $m \geqslant n$, and also

$$
e_{k_{0}-m}=e_{k_{0}-m} \alpha^{k_{0}-m}\left(e_{m}\right) \leqslant e_{k_{0}}, \quad m \geqslant n .
$$

Hence, $c \leqslant 1-e_{k_{0}} \leqslant 1-e_{k_{0}-m}, m \geqslant n$. Thus

$$
\infty \cdot c=\sum_{k=-\infty}^{\infty} e_{k-1}\left(1-\alpha^{-1}\left(e_{k}\right)\right) c=\sum_{k=k_{0}-n+1}^{\infty} e_{k-1}\left(1-\alpha^{-1}\left(e_{k}\right)\right) c .
$$

But, since $1-\alpha^{-1}\left(e_{k}\right)=0$ for $k \geqslant n$,

$$
\infty \cdot c=\sum_{k=k_{0}-n+1}^{n-1} e_{k-1}\left(1-\alpha^{-1}\left(e_{k}\right)\right) c<\infty .
$$

This contradiction shows that $\alpha^{-1}(e) \leqslant e_{k}$ for each $k \in \mathbf{Z}$. Since $\mathscr{B} \neq \mathcal{L}$, there is some $m \in \mathbf{Z}$ with $1-e_{m} \neq 0$. By ergodicity, $\alpha^{-1}(e) \alpha^{-k}\left(1-e_{m}\right) \neq 0$ for some $k>n$. Thus $\alpha^{-k}\left(1-e_{m}\right) e_{m-k} \neq 0\left(\right.$ since $\left.\alpha^{-1}(e) \leqslant e_{m-k}\right)$, and

$$
0 \neq\left(1-e_{m}\right) \alpha^{k}\left(e_{m-k}\right)=\left(1-e_{m}\right) e_{k} \alpha^{k}\left(e_{m-k}\right) \leqslant\left(1-e_{m}\right) e_{m}=0 .
$$

Therefore, $\phi\left(L_{\delta} P L_{\delta}^{*}-P L_{\delta} P L_{\delta}^{*}\right)$ is finite a.e. on $X$.

We now prove that $\phi\left(P-L_{\delta} P L_{\delta}^{*}\right)$ is finite a.e. on $X$. Assume the converse; i.e. $\phi\left(P-L_{\delta} P L_{\delta}^{*}\right) e=\infty \cdot e$ for some projection $e \in \mathcal{L}(Z)$. As shown in the proof for $\phi\left(L_{\delta} P L_{\delta}^{*}-P L_{\delta}^{*}\right)$, it will suffice to show that $e \leqslant e_{k}$ for each $k \in \mathbf{Z}$. We assume $e\left(1-e_{k_{0}}\right) \neq 0$ for some $k_{0} \in \mathbf{Z}$ and

$$
\infty \cdot e=\phi\left(P-L_{\delta} P L_{\delta}^{*}\right) e=\sum_{k=-\infty}^{\infty} e_{k}\left(1-\alpha\left(e_{k-1}\right)\right) e .
$$

Hence,

$$
\begin{aligned}
\infty \cdot e\left(1-e_{k_{0}}\right) & =\sum_{k=-\infty}^{\infty} e_{k}\left(1-\alpha\left(e_{k-1}\right)\right) e\left(1-e_{k_{0}}\right) \\
& =\sum_{k=-n}^{n} e_{k}\left(1-\alpha\left(e_{k-1}\right)\right) e\left(1-e_{k_{0}}\right)<\infty .
\end{aligned}
$$

The contradiction proves that $e \leqslant e_{k}$ for each $k \in \mathbf{Z}$ and completes the proof. 
4. $\mathscr{L}(Z)$ is atomic. Suppose $\alpha$ acts ergodically on $\mathscr{L}(Z)$ and $\mathscr{L}(Z)$ is atomic. We also assume, in this case, that $\alpha^{k}$ is outer for each $k \in \mathbf{Z}$. Since $M$ is finite there is a family $\left\{p_{n}\right\}_{n=1}^{n}$ of mutually orthogonal minimal projections in $\mathcal{L}(Z)$ with $\sum_{n=1}^{n} p_{n}=I$; $\alpha\left(p_{n}\right)=p_{n+1}, n<N$; and $\alpha\left(p_{N}\right)=p_{1}$.

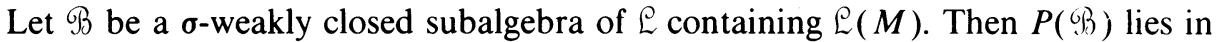
$\{\mathcal{L}(M), \mathscr{R}(M)\}^{\prime}$. But $\{\mathcal{L}(M), \mathscr{R}(M)\}^{\prime}=\left\{\mathcal{L}(Z),\left\{E_{n}\right\}_{n=-\infty}^{\infty}\right\}^{\prime \prime}$ (by Proposition 4.2 of [6]), hence there is a sequence $\left\{e_{k}\right\}_{k=-\infty}^{\infty}$ of projections in $\mathcal{L}(Z)$ such that $P(\mathscr{G})=$ $\sum e_{k} E_{k}$.

Under these assumptions the results of the preceding section hold here. In this case, however, we can say more about the algebras in question.

LEMMA 4.1. Let $f$ be in $Z^{\prime}$ with $\int_{X} f d \nu>0$. Then the algebra $\beta=\varrho^{\beta}[0, \infty)$ of Lemma 3.2 contains $L_{\delta}^{n_{0}} \varrho_{+}$for some integer $n_{0} \geqslant 0$. Consequently, 93 satisfies conditions (i)-(iv).

Proof. Let $c_{n} \in \mathcal{L}(Z)$ be the projections introduced in Lemma 3.2. We can write $f=\sum_{n=1}^{N} \lambda_{n} p_{n}$ where $\lambda_{n} \in \mathbf{R}$ and $\sum_{n=1}^{N} \lambda_{n}>0$; hence $\alpha^{k}(f)=\sum_{n=1}^{N} \lambda_{n} \alpha^{k}\left(p_{n}\right)$ for each $k \in \mathbf{Z}$, and $\sum_{k=0}^{N-1} \alpha^{k}(f)=\left(\sum_{n=1}^{N} \lambda_{n}\right) I>0$. Let $r$ be $\sum_{n=1}^{N} \lambda_{n}$. Then, using the notation introduced in the discussion preceding Lemma 3.2, $f_{N}=r I>0$; thus $c_{N}=1$. Let $\lambda_{0}$ be $\min \left\{\lambda_{n}: 1 \leqslant n \leqslant N\right\}$. Then for some $m_{0} \in \mathbf{Z}_{+}, N \lambda_{0}+r m_{0}>0$. Let $n_{0}$ be $m_{0} N$. Then for $n \geqslant n_{0}, n=m_{1} N+m_{2}$ for some $m_{1} \geqslant m_{0}$ and $0 \leqslant m_{2}<N$; we get

$$
\begin{aligned}
f_{n} & =f_{m_{1} N}+\alpha^{m_{1} N}\left(f_{m_{2}}\right)=m_{1} r+\alpha^{m_{1} N}\left(f_{m_{2}}\right) \\
& \geqslant m_{0} r+\alpha^{m_{1} N}\left(f_{m_{2}}\right) \geqslant m_{0} r+N \lambda_{0}>0 .
\end{aligned}
$$

Hence, for $n \geqslant n_{0}, c_{n}=I$. This implies $L_{\delta}^{n_{0}} \varrho_{+} \subseteq \Re$. Since $f_{n_{0}}>0, f_{-n_{0}}<0$, and it follows that $c_{-n_{0}}=0$, hence $\Re \neq \mathcal{L}$. Thus we can use Corollary 3.5 to complete the proof.

THEOREM 4.2. Let $f$ lie in $\mathscr{Z}^{\prime}$ and let $\mathscr{B}$ be a $\sigma$-weakly closed subalgebra of $\varrho$ containing $\mathcal{L}^{\beta}[0, \infty)$ (where $\beta$ arises from $f$ as in Lemma 3.2). Then $\beta=\mathcal{L}^{\gamma}[0, \infty)$ for some flow $\gamma$ on $\mathcal{L}$. Moreover, if $\int_{X} f d \nu=0$, then $\mathscr{B}$ is a nest subalgebra.

Proof. Since $\mathscr{B}_{0}=\mathfrak{L}^{\beta}[0, \infty)$ contains $\mathcal{L}(M)$, and $\Re_{0}+\mathscr{B}_{0}^{*}$ is $\sigma$-weakly dense in $\mathcal{L}, \mathscr{B}$ satisfies (i) and (ii).

We now distinguish between two cases.

Case 1. $\int_{X} f d \nu \neq 0$. We can assume $\int_{X} f d \nu>0$ (the other possibility can be handled similarly) and apply Lemma 4.1 to find that, for some $n_{0} \in \mathbf{Z}, L_{\delta}^{n_{0}} \varrho_{+} \subseteq \mathscr{G}_{0}$ $\subseteq \Re$. From this, using Corollary 3.5, it follows that $\tilde{f}=\phi\left(P-L_{\delta} P L_{\delta}^{*} P\right)-$ $\phi\left(L_{\delta} P L_{\delta}^{*}-P L_{\delta} P L_{\delta}^{*}\right)$ lies in $\mathscr{Z}_{\tilde{\alpha}}^{\prime}$ where $P=P\left(\mathscr{乃}_{0}\right)$. By Lemma $3.2, \tilde{f}$ gives rise to a flow $\tilde{\beta}$ and an algebra $\tilde{\mathscr{B}}=\complement^{\tilde{\beta}}[0, \infty)=\left\{T \in \mathcal{L}: \varepsilon_{k}(T) \in \tilde{c}_{k} \mathscr{L}(M) L_{\delta}^{k}\right.$ for each $k \in$ $\mathbf{Z}\}$, where $\hat{\tilde{c}}_{k}=\left\{x \in X: \tilde{f}_{k}(x) \geqslant 0\right\}$. Let $\hat{c}_{k}$ be $\left\{x \in X: f_{k}(x) \geqslant 0\right\}$ and $c_{k} \in \mathcal{L}(Z)$ the corresponding projections in $\mathcal{L}(Z)$ for $k \in \mathbf{Z}$. We now show that $\tilde{c}_{k} \leqslant c_{k}$ and this implies $\tilde{\mathscr{B}} \subseteq \mathscr{G}_{0}$, since $\mathscr{B}_{0}=\left\{T \in \mathcal{L}: \varepsilon_{k}(T) \in c_{k} \mathscr{L}(M) L_{\delta}^{k}\right.$ for each $\left.k \in \mathbf{Z}\right\}$. In fact, let $c$ be the projection $\tilde{c}_{k}\left(1-c_{k}\right)$ and assume $c \neq 0$. Since $c \leqslant \tilde{c}_{k}, \tilde{f}_{k} \geqslant 0$ a.e. on $c$. Lemma 3.3, applied to the algebra $\mathscr{B}_{0}$, shows that for almost every $x \in X$, if 
$\tilde{f}_{k}(x) \geqslant 0$ then $\phi\left(L_{\delta} P L_{\delta}^{*}-P L_{\delta} P L_{\delta}^{*}\right)(x)=0$; hence,

$$
\phi\left(L_{\delta} P L_{\delta}^{*}-P L_{\delta} P L_{\delta}^{*}\right) c=0 .
$$

From the proof of Lemma 3.3, applied to $\mathscr{G}_{0}$, we see that

$$
\phi\left(L_{\delta} P L_{\delta}^{*}-P L_{\delta} P L_{\delta}^{*}\right)\left(1-c_{k}\right)=\phi\left(L_{\delta} P L_{\delta}^{*}-P L_{\delta} P L_{\delta}^{*}\right),
$$

but since $0 \neq c \leqslant 1-c_{k}$, this contradicts $(*)$. This proves $\tilde{c}_{k} \leqslant c_{k}$ and, hence, $\tilde{\mathscr{G}} \subseteq \mathscr{G}_{0} \subseteq \mathscr{G}$. But $\tilde{\mathscr{B}}$ is a maximal subdiagonal algebra in $\varrho$. Indeed,

$$
\begin{aligned}
\tilde{f} & =\phi\left(P-L_{\delta} P L_{\delta}^{*} P\right)-\phi\left(L_{\delta} P L_{\delta}^{*}-P L_{\delta} P L_{\delta}^{*}\right) \\
& =\sum_{k=-\infty}^{\infty} e_{k}\left(1-\alpha\left(e_{k-1}\right)\right)-\sum_{k=-\infty}^{\infty} \alpha\left(e_{k-1}\right)\left(1-e_{k}\right)
\end{aligned}
$$

and

$$
\tilde{f}=\sum_{i=1}^{N} m_{i} p_{i}
$$

where

$$
\begin{aligned}
m_{i}= & \#\left\{k \in \mathbf{Z}: e_{k}\left(1-\alpha\left(e_{k-1}\right)\right) p_{i} \neq 0\right\} \\
& -\#\left\{k \in \mathbf{Z}: \alpha\left(e_{k-1}\right)\left(1-e_{k}\right) p_{i} \neq 0\right\} \in \mathbf{Z} .
\end{aligned}
$$

Hence, $\tilde{\beta}_{t}=\tilde{\beta}_{t+2 \pi k}$ for each $k \in \mathbf{Z}$, and the map $\tilde{\varepsilon}=\int_{0}^{2 \pi} \tilde{\beta}_{t} d t$ (where the integral converges in the $\sigma$-weak operator topology) defines a normal faithful expectation form $\mathcal{L}$ onto $\mathcal{L}^{\tilde{\beta}}(\{0\})=\tilde{\mathscr{\beta}} \cap \tilde{\mathscr{B}}^{*}$ satisfying $\tilde{\varepsilon} \cdot \tilde{\beta} t=\tilde{\varepsilon}$ and making $\tilde{\mathscr{B}}$ a maximal subdiagonal algebra. (See [2, Theorem 3.15].) Now we can use [7, Theorem 1] to conclude that $\mathscr{B}$ satisfies (iii) (with the notation of that theorem $\mathcal{L}$ is $L^{\infty}, \tilde{\mathscr{B}}$ is $H^{\infty}$, and $\mathscr{B}$ is a $\sigma$-weakly closed subspace of $L^{\infty}$ satisfying $H^{\infty} \mathscr{B} \subseteq \mathscr{B}$ ). Thus we can apply Corollary 3.5 to $\mathscr{B}$ to complete the proof in this case.

Case 2. $\int_{X} f d \nu=0$. We have $f=\sum_{n=1}^{N} \lambda_{n} p_{n}$, where $\lambda_{n} \in \mathbf{R}$ and $\sum_{n=1}^{N} \lambda_{n}=0$. Let $d_{n}$ be $\sum_{k=1}^{n} \lambda_{k}$ for $1 \leqslant n \leqslant N$; then $d_{n}-d_{n-1}=\lambda_{n}$ for $n>1$ and $d_{1}-d_{N}=\lambda_{1}$. Let $d$, in $\mathcal{E}(Z)$, be $\sum_{n=1}^{N} d_{n} p_{n}$. Then $d-\alpha(d)=\Sigma d_{n} p_{n}-\sum d_{n} \alpha\left(p_{n}\right)=\Sigma \lambda_{n} p_{n}=f$ and, similarly, $d-\alpha^{k}(d)=f_{k}$ for each $k \in \mathbf{Z}$. Consequently,

$$
\beta_{t}\left(L_{x} L_{\delta}^{k}\right)=\left(\exp i t f_{k}\right) L_{x} L_{\delta}^{k}=\exp i t\left(d-\alpha^{k}(d)\right) L_{x} L_{\delta}^{k}, \quad x \in M, k \in \mathbf{Z} .
$$

But

$$
\exp \left(-i t \alpha^{k}(d)\right) L_{\delta}^{k}=L_{\delta}^{k}(\exp (-i t d))
$$

hence,

$$
\beta_{t}\left(L_{x} L_{\delta}^{k}\right)=\exp (i t d) L_{x} L_{\delta}^{k} \exp (-i t d)
$$

i.e. $\beta_{t}$ is inner for each $t \in \mathbf{R}$.

By [2, Theorem 4.2.3], $\mathscr{G}_{0}$ is a nest subalgebra of $\mathcal{L}$. We shall show that $\mathscr{B}$ is also.

As was seen in Lemma $3.2, \mathscr{B}_{0}$ is determined by the projections $\left\{c_{k}\right\}$ that correspond to the sets $\hat{c}_{k}=\left\{x \in X: f_{k}(x) \geqslant 0\right\}$. Here,

$$
f_{k}=\sum_{n=1}^{N} d_{n} p_{n}-\sum_{n=1}^{N} d_{n} \alpha^{k}\left(p_{n}\right) \text {. }
$$


Hence $c_{k}=\sum_{n \in F_{k}} p_{n}$, where $F_{k}=\left\{n: d_{n} \geqslant d_{m}\right.$ where $\left.\alpha^{k}\left(p_{m}\right)=p_{n}\right\}$. Now, let $b_{n}$ be the number of $k$ 's such that $d_{k} \leqslant d_{n}$; then $F_{k}=\left\{n: b_{n} \geqslant b_{n}\right.$ where $\left.\alpha^{k}\left(p_{m}\right)=p_{n}\right\}$; hence, we can replace $d$ by $b=\sum b_{n} p_{n}$ and still get the same algebra $\Re_{0}$. We denote by $\tilde{\beta}$ the new flow and we have $\tilde{\beta}_{t+2 \pi n}=\tilde{\beta}_{t}$ for each $n \in \mathbf{Z}$ and $0 \leqslant t \leqslant 1$ (since $b_{n}$ are integers). Thus the map $\tilde{\varepsilon}$ defined by $\tilde{\varepsilon}=\int_{0}^{2 \pi} \tilde{\beta}_{t} d t$ (where the integral converges in the $\sigma$-weak operator topology) is a faithful normal expectation onto $\varrho^{\beta}(\{0\})$ satisfying $\tilde{\varepsilon} \cdot \tilde{\beta_{t}}=\tilde{\varepsilon}$ for all $t \in \mathbf{R}$. It makes $\mathscr{B}_{0}$ a maximal subdiagonal algebra in $\mathcal{L}$ (see [2, Theorem 3.15]). Since $\varrho \supseteq \Re \supseteq \Re_{0}$, and $\Re_{0}$ is a maximal subdiagonal algebra in $\mathcal{L}$, we can use [7, Theorem 1] to conclude that $\mathscr{B}$ satisfies (iii). Thus, there is a sequence $\left\{e_{k}\right\}_{k=-\infty}^{\infty}$ of projections in $\mathcal{L}(Z)$ such that $P(\mathscr{B})=\sum e_{k} E_{k}$ (see Lemma 3.1).

Recall that $\mathscr{O}_{0}$ is determined by $\left\{c_{k}\right\}$ where $\hat{c}_{k}=\left\{x \in X: f_{k}(x) \geqslant 0\right\}$. Since $f_{N} \equiv 0, c_{k N}=1$ for each $k$ in $\mathbf{Z}$. Since $\Re \supseteq \mathscr{B}_{0}, e_{k N}=1$ for each $k \in \mathbf{Z}$. Also, for $m, k \in \mathbf{Z}$.

$$
e_{m+k N}=e_{-k N} \alpha^{-k N}\left(e_{m+k N}\right) \leqslant e_{m}=e_{k N} \alpha^{k N}\left(e_{m}\right) \leqslant e_{m+k N}
$$

hence, $e_{m}=e_{m+k N}$.

For each $1 \leqslant m \leqslant N$, let $q_{m}$ be the projection $\sum_{k=0}^{N-1} \alpha^{k}\left(p_{m}\right) e_{k}$. We shall show that

$$
\mathscr{B}=\mathcal{L} \cap \operatorname{alg}\left\{q_{m}: 1 \leqslant m \leqslant N\right\}
$$

hence, $\mathscr{B}$ is a nest subalgebra. Denote the right-hand side of $(*)$ by $\tilde{\mathscr{B}}$.

$\tilde{\mathscr{B}} \subseteq \mathscr{B}$. Take $T$ in $\tilde{\mathscr{B}}$, then, for each $k \in \mathbf{Z}, 1 \leqslant m \leqslant N, T$ maps $\alpha^{k}\left(p_{m}\right) e_{k} E_{k}\left(L^{2}\right)$ into $q_{m} E_{k}\left(L^{2}\right)$. Since $T \in \mathcal{L}$, and $\sum_{n=-\infty}^{\infty} \alpha^{n}\left(p_{m}\right) E_{n}$ is the projection $R_{p_{m}}$ in $\mathcal{R}\left(=L^{\prime}\right)$, $T$ maps $\alpha^{k}\left(p_{m}\right) e_{k} E_{k}\left(L^{2}\right)$ into $\sum_{n=-\infty}^{\infty} \alpha^{n}\left(p_{m}\right) E_{n}\left(L^{2}\right)$. But

$$
\begin{aligned}
q_{m} \sum_{n=-\infty}^{\infty} \alpha^{n}\left(p_{m}\right) E_{n} & =\sum_{j=0}^{N-1} e_{j} \alpha^{j}\left(p_{m}\right) \sum_{n=-\infty}^{\infty} \alpha^{n}\left(p_{m}\right) E_{n} \\
& =\sum_{n=-\infty}^{\infty} \alpha^{n}\left(p_{m}\right) e_{n} E_{n} \leqslant \sum_{n=-\infty}^{\infty} e_{n} E_{n}=P(\Re) .
\end{aligned}
$$

Since

$$
\sum_{k=-\infty}^{\infty} \sum_{m=1}^{N} \alpha^{k}\left(p_{m}\right) e_{k} E_{k}=\sum_{n=-\infty}^{\infty} e_{n} E_{n}=P(\Re),
$$

$T$ maps $P(\Re)\left(L^{2}\right)=[\Re]_{2}$ into itself. In particular, $T \psi \in[\Re]_{2}$ and, using condition (iii), $T$ lies in $\mathscr{B}$.

$\mathscr{B} \subseteq \tilde{\mathscr{B}}$. Fix $T \in \mathscr{B}$ and $0 \leqslant k \leqslant N-1$. Since $T$ maps $e_{k} E_{k}\left(L^{2}\right)$ into $\sum_{j=-\infty}^{\infty} e_{j} E_{j}\left(L^{2}\right)$, it maps $\alpha^{k}\left(p_{m}\right) e_{k} E_{k}\left(L^{2}\right)$ into $\sum_{j=-\infty}^{\infty} e_{j} E_{j}\left(L^{2}\right)$ for each $1 \leqslant m \leqslant N$. It also maps it into $\sum_{n=-\infty}^{\infty} \alpha^{n}\left(p_{m}\right) E_{n}\left(L^{2}\right)=R_{p_{m}}\left(L^{2}\right)$ since $T \in \mathcal{L}$. But

$$
\left(\sum e_{j} E_{j}\right)\left(\sum \alpha^{n}\left(p_{m}\right) E_{n}\right)=\sum e_{n} \alpha^{n}\left(p_{m}\right) E_{n} \leqslant q_{m} ;
$$

hence, $T$ maps $\alpha^{k}\left(p_{m}\right) e_{k} E_{k}\left(L^{2}\right)$ into $q_{m}\left(L^{2}\right)$.

For $n \in \mathbf{Z}, \alpha^{k}\left(p_{m}\right) e_{k} E_{n}=R_{\delta}^{n-k} \alpha^{k}\left(p_{m}\right) e_{k} E_{k}$ and

$$
T R_{\delta}^{n-k} \alpha^{k}\left(p_{m}\right) e_{k} E_{k}\left(L^{2}\right)=R_{\delta}^{n-k} T \alpha^{k}\left(p_{m}\right) e_{k} E_{k}\left(L^{2}\right) \subseteq R_{\delta}^{n-k} q_{m}\left(L^{2}\right) \subseteq q_{m}\left(L^{2}\right) \text {. }
$$


Hence, $T$ maps $\alpha^{k}\left(p_{m}\right) e_{k} E_{n}\left(L^{2}\right)$ into $q_{m}\left(L^{2}\right)$ for each $m, n \in \mathbf{Z}, 1 \leqslant m \leqslant N$. Since

$$
\sum_{n=-\infty}^{\infty} \alpha^{k}\left(p_{m}\right) e_{k} E_{n}=\alpha^{k}\left(p_{m}\right) e_{k} \quad \text { and } q_{m}=\sum_{k=0}^{N-1} \alpha^{k}\left(p_{m}\right) e_{k},
$$

$T$ maps $q_{n}\left(L^{2}\right)$ into $q_{n}\left(L^{2}\right)$. Hence $T \in \mathscr{G}$.

Hence, $\mathscr{G}=\tilde{\mathscr{B}}$, and $\mathscr{B}$ is a nest subalgebra. The fact that $\mathscr{B}$ is $\mathfrak{L}^{\gamma}[0, \infty)$ for some flow $\gamma$ on $\mathcal{L}$ follows from [2, Theorem 4.23].

\section{BIBLIOGRAPHY}

1. J. Dixmier, Les algèbres d'opérateurs dans l'espace Hilbertien, Gauthier-Villars, Paris, 1969.

2. R. I. Loebl and P. S. Muhly, Analyticity and flows in von Neumann algebras, J. Funct. Anal. 29 (1978), 214-252.

3. G. Mackey, Point realizations of transformation groups, Illinois J. Math. 6 (1962), 327-335.

4. M. McAsey, P. S. Muhly and K.-S. Saito, Nonselfadjoint crossed products (invariant subspaces and maximality), Trans. Amer. Math. Soc. 248 (1978), 381-409.

5. Nonselfadjoint crossed products. II, J. Math. Soc. Japan. 33 (1981), 485-495.

6. K.-S. Saito, Automorphisms and nonselfadjoint crossed products, preprint.

7. . Invariant subspaces for finite maximal subdiagonal algebras, Pacific. J. Math. 93 (1981), $431-434$.

8. I. E. Segal, A non-commutative extension of abstract integration, Ann. of Math. (2) 57 (1953), 401-457.

9. B. Solel, The invariant subspace structure of nonselfadjoint crossed product, Trans. Amer. Math. Soc. (to appear).

10. M. Takesaki, Duality for crossed products and the structure of von Neumann algebras of type III, Acta Math. 131 (1973), 249-310.

School of Mathematical Sciences, Tel-Aviv University, Tel-Aviv, IsRael

Current address: Department of Mathematics, Statistics and Computing Science, Dalhousie University, Halifax, Nova Scotia, Canada 